\title{
Combined Coding and Shaping over a Multitone Channel
}

\author{
A. K. Khandani ${ }^{1,2}$, P. Kabal ${ }^{1,2}$ and H. Leib ${ }^{1}$ \\ ${ }^{1}$ McGill University, 3480 University, Montreal, Canada, H3A-2A7 \\ ${ }^{2}$ INRS-Telecommunications, 3 Place du Commerce, Verdun, Canada, H3E-1H6
}

\begin{abstract}
In this work, the idea of combined shaping and coding of a signal constellation over a multitone channel is introduced. In selecting such a constellation, we are faced with the problems of distributing the rate and the energy among the subchannels. Assuming continuous approximation, these factors can be selected independently. However, in the discrete case, one obtains a better performance by using a joint optimization procedure. More importantly, often the structure of the constellation boundary imposes some restrictions on the rate distribution. This provides a stronger coupling between these factors. We introduce two joint optimization methods, partly integer, for distributing the rate and the energy. In the first method, the minimum distance to noise ratio (protection) along all the dimensions is the same. The proposed method maximizes this protection. In the second method, this restriction is relaxed. In this case, the average error probability is minimized. Neither of these methods has a higher complexity than the conventional schemes. The second method outperforms the first one. As part of the calculations, we have found a closed form formula for the weight distribution of the scaled $\mathbf{E}_{8}$ lattice.
\end{abstract}

\section{Introduction}

An orthonormal set of $M$ modulating signals divide a channel into $M$ one-dimensional subchannels. In general, the subchannels have both time and frequency overlap. The channel noise is assumed to be additive Gaussian of unity power. The set of the modulator, channel and demodulator is normalized to provide unity gain in each of the subchannels. This is equivalent to $M$ one-dimensional subchannels with additive Gaussian noise of powers $\sigma_{i}^{2}, i=0, \ldots, M-1$. In general, if the original noise is colored or the channel is nonflat, the $\sigma_{i}$ 's will be different.

In selecting an $N$-dimensional constellation, $N \leq M$, over this channel, we should select a finite set of $N$ dimensional points bounded within a shaping region $\mathcal{R}_{N}$. In continuous approximation, the distribution of points is approximated by a continuous uniform density within $\mathcal{R}_{N}$. In this case to have equal minimum distance along different dimensions at the channel output, the volume of the Voronoi region at the channel input should be proportional to $\prod \sigma_{i}$, where the product is over the set of the constellation dimensions. The ratio of the volume of $\mathcal{R}_{N}, V\left(\mathcal{R}_{N}\right)$, and $\prod \sigma_{i}$ determines the rate. The second moment of $\mathcal{R}_{N}$ $I\left(\mathcal{R}_{N}\right)$, determines the energy. The design objective is to minimize the probability of error for a given rate and energy. The optimum point is achieved by leaving $M-N$ of the subchannels empty. The number of empty subchannels is affected by two conflicting phenomena: (i) A larger number of empty subchannels decreases $\Pi \sigma_{i}$. (ii) For a fixed second moment, decreasing the dimensionality results in a lower $V\left(\mathcal{R}_{N}\right)$. For a fixed $\prod \sigma_{i}$, fixed rate and fixed energy, the volume of the Voronoi region and consequently the probability of error is determined by $V\left(\mathcal{R}_{N}\right)$. The structure of the regions which maximize $V\left(\mathcal{R}_{N}\right)$ for a given $I\left(\mathcal{R}_{N}\right)$ is introduced in [1]. These regions have equal second moments along different dimensions. This results in an equal distribution of energy. In this case, the rate is (independently) distributed such that the minimum distance to noise ratio along all the dimensions is the same. This is a special case of a more general result that in continuous approximation coding and shaping are independent. In other words, as far as coding is concerned, the constellation is assumed to be an infinite array of points without boundary and as far as shaping is concerned, it is assumed that there are infinite points within the shaping region. In this case, the performance measure has independent factors which can be optimized separately.

However, in the discrete case these factors are coupled and one gains by using a joint optimization procedure. The first coupling is due to the fact that in a constellation with finite cardinality, rate is a discrete quantity. A stronger coupling is produced by the addressing scheme. Addressing is the assignment of the data bits to the constellation points. The addressing complexity is a major attribute of the shaping regions. To obtain regions with tractable complexity, one should impose restrictions on the rate distribution. For example, in this work the number of the points in each two-dimensional subconstellation is of the form $2^{R_{j}}(1+2 / N)$ where $N$ (dimensionality) is an integral power of two and the $R$, 's are integer numbers greater than or equal to $\log _{2}(N / 2)$.

In the proposed methods these two factors are jointly selected to minimize the probability of error. In the first method, the minimum distance to noise ratio along all the dimensions is the same. In the second method, this restriction is relaxed. This freedom is used to reduce the effective number of the nearest neighbors of the coding lattice.

\subsection{1}


Neither of these methods increases the complexity over the conventional schemes. The second method outperforms the first one.

\section{Boundary shaping}

Shaping is based on a modified version of the method introduced in [2] and generalized in [3]. In our case there are different number of points in different two-dimensional subconstellations. To transmit $R$ bits per two dimensions in an $N=2 n$-dimensional TCM (Trellis Coded Modulation) scheme with one bit redundancy, a signal constellation with $2^{n R+1}$ points is needed. Assume that the rate allocated to the $j$ 'th subspace is $R_{j}$. To construct the constellation, the two-dimensional subconstellations are divided into an inner group and an outer group. The number of points in the inner group of the $j^{\text {'th }}$ subconstellation is equal to $2^{R_{j}}$. The integer numbers $R_{j}$ satisfy $\sum_{j} R_{j}=n R$. The number of points in the outer group is $1 / n$ of that in the inner group. This is possible if $n$ is a power of two and $R_{j} \geq \log _{2}(n)$. The inner group is selected as the cross constellation with rate $R_{j}$, [4]. The outer points are selected as a subset of the cross constellation with rate $R_{j}+1$ located around the inner group and with the least energy. Both the inner and the outer group have four way symmetry and contain an equal number of points from each partition of an Ungerboeck partition chain. These are important considerations in a practical implementation, [2].

The $N$-dimensional constellation is constructed by concatenating $n$ such two-dimensional subconstellations and excluding the $N$-dimensional points corresponding to more than one two-dimensional outer point. Addressing is achieved by a lookup table with $\left(1+n \log _{2} n\right)$ input lines and $n\left\lceil\log _{2}(1+n)\right\rceil$ output lines.

The Constellation-Expansion-Ratio (CER) of a shaping region is defined as the ratio of the number of points per two dimensions to the minimum necessary number of points per two dimensions, [3]. For the region under consideration, CER is equal to $(1+1 / n) \times 2^{-(1 / n)}$.

For each two-dimensional subconstellation, the inner group is used $N-1$ times as often as the outer group. This means that the average energy per two dimensions is equal to $(N-1) / N$ times the average energy of the inner group plus $1 / N$ times the average energy of the outer group. Table 1 shows the average energy per two dimensions $(A)$ for $N=8$ and for a minimum distance of one as a function of the rate per two dimensions $(R)$. Column $A_{c}(R)$ is the energy obtained by applying continuous approximation to a rectangular region, $A_{c}(R)=2^{(R+0.25)} / 6$.

\section{Coding}

The points of the two dimensional subconstellations belong to the half integer lattice (cross constellation, [4]). Different two-dimensional subconstellations are scaled with different scale factors. Consequently, the $N$-dimensional points belong to a scaled version of the $N$-dimensional half integer

\begin{tabular}{rrr}
\hline$R$ & $A(R)$ & $A_{c}(R)$ \\
\hline 4 & 3.0 & 3.2 \\
5 & 5.9 & 6.3 \\
6 & 11.9 & 12.7 \\
7 & 23.6 & 25.4 \\
8 & 47.8 & 50.7 \\
9 & 94.6 & 101.5 \\
\hline
\end{tabular}

Table 1 Average energy per two dimensions as a function of the rate for a minimum distance of one, $N=8$.

lattice. This lattice is partitioned into the cosets of a sublattice. The unscaled version of this sublattice is denoted as the baseline lattice. It should be mentioned that scaling does not change the group property of the lattices. Consequently, the coset decomposition is the same as in the unscaled lattices and the cosets are congruent to each other. Scaling also preserves the distance invariance property, i.e., from every point of the scaled lattice the surrounding points look the same. In each signaling interval some of the data bits are encoded and used to select one of the cosets. The rest of the data bits select a point within the selected coset. We assume that the dominant error event is the error within a coset. This is the case in most of the TCM schemes. The numerical examples are based on dimensionality eight and the baseline lattice $\mathbf{E}_{8},[5]$.

\section{3..1 Weight distribution of the scaled lat- tices}

The weight distribution of a set of points $\Lambda$ with respect to a given center is defined as, [5],

$$
\Theta_{\Lambda}(q)=\sum_{u \in \Lambda} q^{\|u\|^{2}}=\sum_{x} N(x) q^{x},
$$

where $\|u\|$ is the norm of vector associated with point $u$ and $N(x)$ is the number of the points at square distance $x$ from the center. For a set of points with the distance invariance property, the weight distribution function is independent of the center. This is the case for a scaled lattice.

We assume that the square minimum distance along the $j$ 'th two-dimensional subconstellation $(j \in[0, N / 2-1])$ is equal to $D_{j}$. Using the trellis diagram of the lattice based on the squaring construction [6], we calculate the weight distribution of the scaled lattice $\mathbf{E}_{8}$. The result is,

$$
\begin{gathered}
\Theta_{\mathbf{E}_{\mathbf{s}}}(q)= \\
\theta_{3}^{2}\left(q_{0}\right) \theta_{3}^{2}\left(q_{1}\right) \theta_{3}^{2}\left(q_{2}\right) \theta_{3}^{2}\left(q_{3}\right)+\theta_{3}^{2}\left(q_{0}\right) \theta_{3}^{2}\left(q_{1}\right) \theta_{2}^{2}\left(q_{2}\right) \theta_{2}^{2}\left(q_{3}\right)+ \\
\theta_{3}^{2}\left(q_{0}\right) \theta_{2}^{2}\left(q_{1}\right) \theta_{3}^{2}\left(q_{2}\right) \theta_{2}^{2}\left(q_{3}\right)+\theta_{3}^{2}\left(q_{0}\right) \theta_{2}^{2}\left(q_{1}\right) \theta_{2}^{2}\left(q_{2}\right) \theta_{3}^{2}\left(q_{3}\right)+ \\
\theta_{2}^{2}\left(q_{0}\right) \theta_{3}^{2}\left(q_{1}\right) \theta_{3}^{2}\left(q_{2}\right) \theta_{2}^{2}\left(q_{3}\right)+\theta_{2}^{2}\left(q_{0}\right) \theta_{3}^{2}\left(q_{1}\right) \theta_{2}^{2}\left(q_{2}\right) \theta_{3}^{2}\left(q_{3}\right)+ \\
\theta_{2}^{2}\left(q_{0}\right) \theta_{2}^{2}\left(q_{1}\right) \theta_{3}^{2}\left(q_{2}\right) \theta_{3}^{2}\left(q_{3}\right)+\theta_{2}^{2}\left(q_{0}\right) \theta_{2}^{2}\left(q_{1}\right) \theta_{2}^{2}\left(q_{2}\right) \theta_{2}^{2}\left(q_{3}\right)+ \\
8 \theta_{2}\left(q_{0}\right) \theta_{2}\left(q_{1}\right) \theta_{2}\left(q_{2}\right) \theta_{2}\left(q_{3}\right) \theta_{3}\left(q_{0}\right) \theta_{3}\left(q_{1}\right) \theta_{3}\left(q_{2}\right) \theta_{3}\left(q_{3}\right),
\end{gathered}
$$

where,

$$
q_{j}=q^{4 D_{j}}, \quad j=0,1,2,3,
$$

and $\theta_{2}$ and $\theta_{3}$ are the Jacobi theta functions, [5]. This expression will be used in calculating the error probability. 


\section{Probability of error}

For an additive Gaussian noise of power $\sigma^{2}$, the probability of error between two points with distance $d$ is upper bounded by,

$$
P_{e} \leq \frac{1}{\sqrt{2 \pi}} \exp \left(-d^{2} / 2 \sigma^{2}\right)
$$

Substituting (3) in the union bound results in an upper bound for error probability. This bound can be calculated by substituting $q=\exp \left[-1 /\left(2 \sigma^{2}\right)\right]$ in $\Theta_{\Lambda}-1$, where $\Theta_{\Lambda}$ is given in (1), and multiplying the result by $1 / \sqrt{2 \pi}$. In practice, we truncate the weight distribution to the set of the nearest neighbors. This is based on the assumption that after scaling, the points in the second shell do not come nearer to the center than the points in the first shell. For the lattice $\mathbf{E}_{8}$, truncating (2) to the set of the 240 nearest neighbors, results in,

$$
\begin{aligned}
P_{e} \simeq & \frac{4}{\sqrt{2 \pi}} \times \\
\left(Z_{0}^{4}+\right. & Z_{1}^{4}+Z_{2}^{4}+Z_{3}^{4}+4 Z_{0}^{2} Z_{1}^{2}+4 Z_{0}^{2} Z_{2}^{2}+4 Z_{0}^{2} Z_{3}^{2}+ \\
& \left.4 Z_{1}^{2} Z_{2}^{2}+4 Z_{1}^{2} Z_{3}^{2}+4 Z_{2}^{2} Z_{3}^{2}+32 Z_{0} Z_{1} Z_{2} Z_{3}\right),
\end{aligned}
$$

where $Z_{j}=\exp \left(-D_{j} / 2 N_{j}\right)$ and $N_{j}, D_{j}$ are the noise power and the square minimum distance in the $j$ 'th twodimensional subspace. We use the notation $P_{e} \simeq F\left(Z_{j}, j=\right.$ $0,1,2,3)$ to show the function in (4).

\section{Problem statement}

We have an $M_{0}$-dimensional multitone channel. The available energy is equal to $E_{0}$. We use a Fourier basis for modulation. We leave $M_{0}-N_{0}$ of the dimensions empty. In the case that $M_{0}$ is odd, the dimension with the highest noise power is always empty. The noise powers of the remaining dimensions are pairwise equal. The two dimensions with the equal noise power constitute a (two-dimensional) subchannel. From now on the word subchannel refers to a twodimensional subchannel. The nonempty subchannels are indexed by $i \in\left[0, \ldots, n_{0}-1\right], n_{0}=N_{0} / 2$. These are divided into $K$ groups each of $N$ dimensions. A two-dimensional subconstellation is employed over each subchannel. This results in an $N$-dimensional subconstellation over each group. Each group uses an independent TCM scheme. The total rate is equal to $R_{0}=n_{0} R+K$ corresponding to $R$ bits per each nonempty subchannel and one bit redundancy for each coding group. The two-dimensional subconstellations are indexed by $(k, j)$ where $k \in[0, K-1]$ is the index of the group and $j \in[0, n-1], n=N / 2$ is the index within the group. The noise power, the square minimum distance and the minimum distance to noise ratio (protection) of the $(k, j)$ 'th two-dimensional subconstellation are shown by, $N_{j}^{k}, D_{j}^{k}$ and $P_{j}^{k}=D_{j}^{k} / N_{j}^{k}$, respectively. The corresponding rate and energy are related by,

$$
E_{j}^{k}=A\left(R_{j}^{k}\right) \times D_{j}^{k}
$$

where $A(R)$ is given in Table 1 .

The total gain of the system, $\gamma_{t}$, is defined as the saving in energy with respect to a reference system with the same probability of error. The reference system uses an $M_{0^{-}}$ dimensional flat channel with unity gain and is composed of the points of the cubic lattice (no coding) bounded within a hypercube (no shaping). For the reference system,, we assume continuous approximation and use (3) for the error probability. Equating the error probabilities, $\gamma_{t}$ is equal to the ratio of the energies. This results in,

$$
\gamma_{t}=\frac{2^{Q} M_{0}}{6 E_{0}} \log \left(\frac{1}{\sqrt{2 \pi} P_{e}}\right)
$$

where $Q$ is the data rate per two dimensions of the reference system, $Q=N_{0} R / M_{0}$. The $\gamma_{t}$ reflects (i) the shaping gain, (ii) the coding gain, and (iii) the degradation caused by the nonflat transfer function of the channel. This degradation is due to a loss in dimensionality and/or having $\Pi \sigma_{i}$ greater than one. It can be shown that in continuous approximation, $\gamma_{t}$ is the product of three factors, each representing one of these effects.

We are looking for $R_{j}^{k}$ 's, $E_{j}^{k}$ 's and a rule for grouping the subchannels. This is expressed in terms of the one-toone assignment $(j, k) \Longleftrightarrow i$, where $(j, k)$ is the index of the two-dimensional subconstellation and $i$ is the index of the two-dimensional subchannel. The objective is to maximize $\gamma_{t}$ or equivalently to minimize $P_{e}$. As $R$ is an integer, it is usually impossible to change $N_{0}$ while keeping the total data rate, $N_{0} R / 2$, constant. As a result different systems obtained by changing $N_{0}$ can not be easliy compared. This means that $N_{0}$ should be considered as a fixed parameter of the scheme and then the whole scheme can be compared with other possibilities to make the final selection.

\section{First method: Equal protection along the subchannels}

For equal protection, we set $P_{j}^{k}=P_{0}, \forall j, k$. In this case the optimization problem is formulated as:

$$
\begin{cases}\text { Maximize } & P_{0} \\ \text { Subject to: } & \sum_{k=0}^{K-1} \sum_{j=0}^{n-1} R_{j}^{k}=n_{0} R, \quad R_{j}^{k} \in \mathrm{N}, R_{j}^{k} \geq \log _{2}(n) \\ & \sum_{k=0}^{K-1} \sum_{j=0}^{n-1} E_{j}^{k}=E_{0}, \quad E_{j}^{k} \geq 0,\end{cases}
$$

where $\mathbf{N}$ is the set of integers. Combining (5) and (7) results in,

$$
P_{0}=\frac{E_{0}}{\sum_{k=0}^{K-1} \sum_{j=0}^{n-1} A\left(R_{j}^{k}\right) N_{j}^{k}} .
$$


Using (8) the optimization problem in (7) reduces to,

$$
\begin{cases}\text { Minimize } & \sum_{k=0}^{K-1} \sum_{j=0}^{n-1} A\left(R_{j}^{k}\right) N_{j}^{k} \\ \text { Subject to: } & \sum_{k=0}^{K-1} \sum_{j=0}^{n-1} R_{j}^{k}=n_{0} R, \quad R_{j}^{k} \in \mathrm{N}, R_{j}^{k} \geq \log _{2}(n) .\end{cases}
$$

In this case the assignment $(j, k) \Longleftrightarrow i$ is arbitrary. This problem is solved by the following algorithm:

1. Set $R_{j}^{k}=\log _{2}(n), \forall j, k, n_{0}[R-\log (n)]$ bits remain to be distributed.

2. Allocate one bit to the two-dimensional subconstellation with the least value of $\left[A\left(R_{j}^{k}+1\right)-A\left(R_{j}^{k}\right)\right] N_{j}^{k}$. Update the rates. If there are still bits to be distributed go to step 2 , otherwise quit.

\section{Second method: Nonequal protection along the subchannels}

In this case we minimize the average error probability of the whole system. This is formulated as,

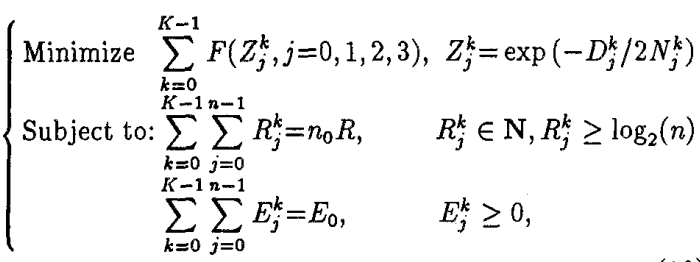

where $D_{j}^{k}$ is related to $E_{j}^{k}$ and $R_{j}^{k}$ by (5). The rate/energy distribution and the assignment rule $(j, k) \Longleftrightarrow i$ are determined by a two step iterative procedure. The first step itself is another iterative procedure and finds the optimum rate distribution for a given energy distribution and vice versa. As the starting point we use the answer obtained by applying the first method. In the second step, we find the optimum assignment rule $(j, k) \Longleftrightarrow i$ for the final answer of the first step. Then the two steps repeat.

\section{7..1 First step}

\section{7..1.1 Optimum rate distribution for a given energy distribution}

Following algorithm is used to find the rate distribution:

1. Set $R_{j}^{k}=\log _{2}(n), \forall j, k . n_{0}[R-\log (n)]$ bits remain to be distributed.

2. Arrange the two-dimensional subconstellations according to the value of $E_{j}^{k} / A\left(R_{j}^{k}\right) N_{j}^{k}$ (protection) in the decreasing order and index them with $i_{1} \in\left[0 ; n_{0}-1\right]$.

3. Arrange the two-dimensional subconstellations according to the value of $E_{j}^{k} / A\left(R_{j}^{k}+1\right) N_{j}^{k}$ in decreasing order and index them with $i_{2} \in\left[0, n_{0}-1\right]$.
4. Find the smallest integer $m \leq n_{0}$ such that for $i_{1}, i_{2} \in$ $[0, m-1]$, the elements in the set indexed by $i_{1}$ are obtained by the permutation of the elements in the set indexed by $i_{2}$. These two-dimensional subconstellations are the candidates for receiving the bit.

5. Allocate one bit to the candidate which by receiving it will result in the least increase in the objective function. Update the rates. If there are still bits to be distributed go to step 2, otherwise quit.

\section{7..1.2 Optimum Energy Distribution for a Given Rate Distribution}

The objective function in (10) is a convex $U$ function of $E_{j}^{k}$ 's. As a result, the global optimum point over the convex region determined by the energy constraint is determined by the Lagrange method. This results in the following set of equations for $E_{j}^{k}$ 's.

$$
\left\{\begin{array}{l}
\frac{\partial}{\partial Z_{j}^{k}}\left[F\left(Z_{j}^{k}, j=0,1,2,3\right)\right]=\lambda N_{j}^{k} A\left(R_{j}^{k}\right) \\
\sum_{k=0}^{K-1} \sum_{j=0}^{n-1} E_{j}^{k}=E_{0} .
\end{array}\right.
$$

This set is solved by an iterative method.

\section{7..2 Second step: Assignment $(j, k) \Longleftrightarrow i$}

This problem is solved by the following algorithm:

1. Arrange the nonempty subchannels according to the value of the noise power in increasing order and index them by $i_{1} \in\left[0, n_{0}-1\right]$.

2. Arrange the two-dimensional subconstellations according to the value of $P_{j}^{k}$ (protection) in the increasing order and index them by $i_{2} \in\left[0, n_{0}-1\right]$.

3. Assign the members of the two sets with the same index to each other.

\section{7..3 Special cases}

We can show that if there exists a rate distribution such that $A\left(R_{j}^{k}\right) N_{j}^{k}=$ constant, then this is optimum for the energy distribution $E_{j}^{k}=E_{0} / n_{0}$ and vice versa. This results in equal protection. Another special case arises when in a given energy updating step we obtain $E_{j}^{k}=E_{0} / n_{0}$. In this case if the total rate is a multiple of $M_{0}$, the optimum rate distribution will be of the form $R_{j}^{k}=R$. The converse is true if the noise powers along different dimensions are equal.

\section{Example}

In this example, we consider a $(1-D)$ partial response channel. The total number of dimensions is equal to $M_{0}=27$ and $N_{0}=24$ dimensions are nonempty. A zero is transmitted between successive $M_{0}$-dimensional blocks. This brings the 
Iannel to zero state at the beginning of each block. We have $R=2$ corresponding to a total data rate of $N_{0} R / 2=24$ bits. There are $K=3$ coding groups each of dimensionality $N=8$. Lattice $\mathbf{E}_{8}$ is used as the baseline lattice. Over a flat channel, the corresponding TCM scheme results in 5.41 $\mathrm{dB}$ coding gain plus the shaping gain of the constellation boundary, [2]. We apply both of our design methods to this problem. The performance is measured in terms of the total gain, $\gamma_{t}$, and the probability of error. Figures (1) and (2) show these parameters as a function of the energy per dimension, $E_{0} / 24$.

Referring to Fig. (1), we note that for low energy per dimension the total gain decreases. This is due to the large number of the nearest neighbors. Referring to Fig. 2, we note that the improvement of the second method is almost equivalent to multiplying the probability of error by a constant factor. This can be considered as reducing the number of the nearest neighbors to some smaller effective value. A justification of this phenomenon is obtained by referring to (4). This equation is composed of the sum of $4 \times 60=240$ terms. Each term corresponds to one of the nearest neighbors of the lattice $\mathbf{E}_{\mathbf{8}}$. It is seen that the distance from the center to $1 / 15$ of the neighbors is determined by the protection along only one two-dimensional subconstellation. The distance to $6 / 15$ of them is determined by the sum of the protections along two of the two-dimensional subconstellations. Finally the distance to $8 / 15$ of the neighbors is determined by the sum of the protections along all the twodimensional subconstellations. When the protections are added it makes no difference which subconstellation has a larger effect on the sum. This flexibility is used by the optimization algorithm to reduce the effective number of the nearest neighbors. We note that for lattices like the Leech lattice with 196560 nearest neighbors, [5], this improvement will be more pronounced.

Summary and conclusions: We have designed a TCM coding scheme for signaling over a multitone channel. This is based on jointly selecting the internal structure (coding) and the boundary (shaping) of the constellation. Instead of dealing with the rate as a continuous variable and rounding the result, we have used an integer optimization procedure for the rate allocation. Two different schemes are proposed. The first scheme has equal minimum distance to noise ratio along all the dimensions. In the second scheme this constraint is removed. The second method outperforms the first one. Neither of the two schemes has higher complexity than the conventional methods. As part of the calculations, we have generalized the concept of the weight distribution to the scaled lattices and found a closed form formula for the weight distribution of the scaled $\mathbf{E}_{8}$ lattice.

\section{References}

[1] A. K. Khandani and P. Kabal, "Shaping multidimensional signal constellations," IEEE Int. Symp. In form. Theory, p. 4, June 1991, Budapest, Hungary.

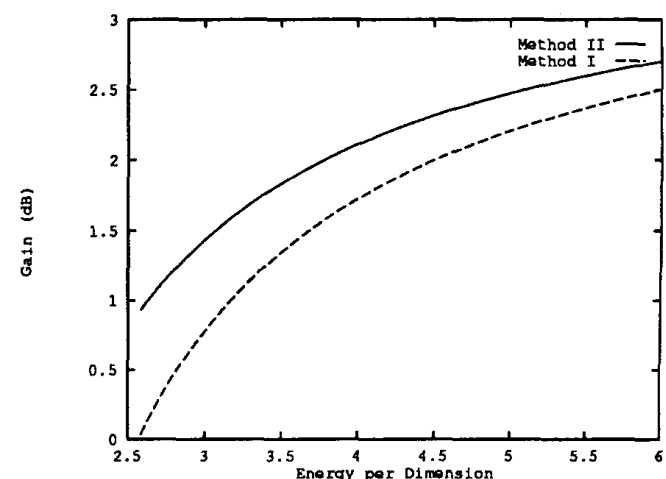

Fig. 1 Total gain as a function of the energy per dimen$\operatorname{sion}\left(E_{0} / N_{0}\right)$ for $M_{0}=27, N_{0}=24, R=2$.

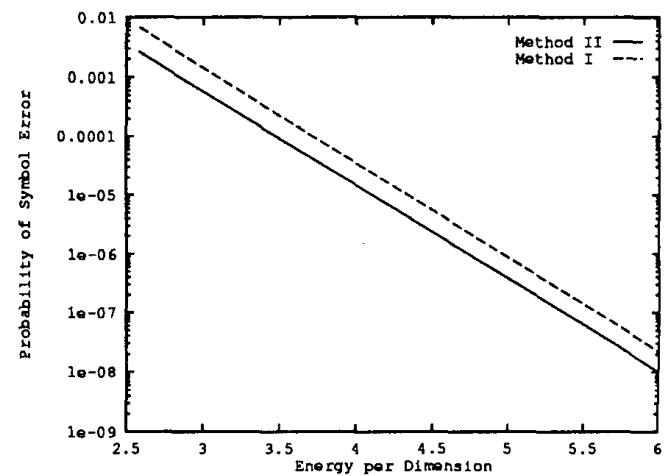

Fig. 2 Probability of symbol error as a function of the energy per dimension $\left(E_{0} / N_{0}\right)$ for $M_{0}=27, N_{0}=24, R=2$.

[2] L. F. Wei, "Trellis coded modulation with multidimensional constellations," IEEE Trans. Inform. Theory, vol. IT-33, pp. 483-501, July 1987.

[3] G. D. Forney, Jr., "Multidimensional constellationsPart I: Introduction, Figures of merit, and Generalized Cross Constellations," IEEE J. Select. Areas Commun., vol. SAC-7, pp. 877-892, August 1989.

[4] G. D. Forney, Jr., R. G. Gallager, G. R. Lang, F. M. Longstaff, and S. U. Quereshi, "Efficient modulation for bandlimited channels," IEEE J. Select. Areas Commun., vol. SAC-2, pp.632-647, 1984.

[5] J. H. Conway and N. J. A. Sloane, Sphere packings, Lattices and groups, Springer-Verlag, 1988.

[6] G. D. Forney, "Coset codes-Part II: Binary lattices and related codes," IEEE Trans. Inform. Theory, vol. IT-34, pp. 1152-1187, September 1988. 